\title{
L'État-providence suédois comme modèle social productif
}

\author{
\begin{tabular}{l|l} 
& \\
Nathalie Morel & Chercheuse associée à Sciences Po, Centre d'études
\end{tabular} \\ européennes.
}

Mots-clés : politique sociale - investissement social - Suède

Dès les années 1930, la Suède a développé une approche originale de la politique sociale, considérant celle-ci non pas comme un coût mais comme un investissement productif qui permet de concilier efficacité économique et équité sociale. Ainsi, le discours très en vogue aujourd'hui, au niveau européen comme au niveau de l'Organisation de coopération et de développement économiques, autour de la perspective d'investissement social, et qui prône la réorientation des dépenses sociales "passives " vers des dépenses sociales " actives", semble s'inspirer des politiques mises en œuvre dans les pays nordiques, et théorisées tout particulièrement en Suède. Cet article analyse les fondements et le développement de ce modèle social productif suédois de façon à mieux comprendre ce qui fait la spécificité de ce modèle et son succès, mais aussi à éclairer les orientations actuelles au niveau européen autour de la perspective d'investissement social.

La Suède est systématiquement présentée comme l'exemple type du régime d'État-providence socialdémocrate, l'un des trois "régimes » identifiés par Gøsta Esping-Andersen dans son ouvrage, devenu classique, Les trois mondes de l'État-providence (1990). Le pouvoir des forces de gauche (syndicats et parti social-démocrate), le degré important de «démarchandisation» (1) que les politiques sociales engendrent, la forte ambition redistributive, et la dimension très collectiviste du modèle sont les caractéristiques généralement associées à ce régime social-démocrate. Pourtant, une analyse retraçant les fondements et le développement des politiques sociales en Suède montre à quel point I'objectif du modèle n'est pas tant de contrôler ou de remplacer le marché (voir l'idée de " politics against markets » de G. Esping-Andersen, 1985), mais plutôt de le rendre plus efficace, tout en protégeant les travailleurs contre ses pires excès. De même, I'objectif des politiques menées n'est pas tant la redistribution des richesses que la promotion de l'égalité des chances par le biais d'une stratégie de dotation en capabilités (2), visant à tirer vers le haut toute la population. Enfin, selon Henrik Berggren et Lars Trägårdh (2006), la dimension en apparence très collectiviste du modèle serait en fait le fruit d'un individualisme exacerbé et viserait à promouvoir et protéger l'autonomie des individus vis-à-vis de la famille et de la communauté par le biais d'un État fort.

L'originalité de la Suède, que l'on retrouve dans une large mesure dans les autres pays nordiques, est que, dès les années 1930, les politiques sociales qui se sont mises en place ont été conçues comme un investissement productif visant à concilier efficacité économique et équité sociale. Ainsi, le discours très en vogue actuellement au niveau européen comme au niveau de l'Organisation de coopération et de développement économiques (OCDE) (Morel et al., 2012 a ; Mahon, 2013), autour de la perspective $d^{\prime}$ investissement social pour permettre de concilier croissance économique et équité par le biais d'une réorientation des dépenses sociales "passives» vers des dépenses sociales

\footnotetext{
1) Ce concept de "démarchandisation » a été développé par G. Esping-Andersen (1990) pour mesurer le degré d'indépendance des individus vis-à-vis du marché en fonction de l'étendue et de la qualité des droits sociaux dont ils bénéficient. Le niveau de démarchandisation est calculé en regardant les conditions d'accès aux droits sociaux (durée et/ou montant des cotisations nécessaires), du montant des prestations (taux d'indemnisation) et de la durée de versement des prestations.

(2) On reprend ici le concept développé par Amartya Sen, qui désigne la liberté réelle des individus, en interaction avec leur contexte social et institutionnel, de réaliser leurs projets de vie. Comme l'a souligné Jérôme Gautié, la conception d'A. Sen de I'individu et de la société se distingue de celle de John Rawls en ce qu'il souligne qu'il ne suffit pas de doter de façon équitable les individus de " capitaux » au sens large ou, dans la terminologie de J. Rawls, de « biens premiers sociaux ». Pour A. Sen, il faut aussi se soucier des possibilités réelles qu'ont les individus de les mobiliser pour mener à bien leurs projets de vie : ainsi, selon l'exemple souvent avancé, à dotation égale, une personne handicapée ne pourra pas tirer le même parti de ses dotations qu'une personne qui ne l'est pas. De fait, au-delà des «capitaux », il faut se soucier des «capabilités » (capabilities) à les utiliser (Gautié, 2003:29). Dans le contexte suédois, la mise en place de services sociaux universels vise ainsi à fournir ces capabilités.
} 
« actives", semble s'inspirer des politiques mises en œuvre dans les pays nordiques, et théorisées tout particulièrement en Suède. II paraît donc intéressant d'analyser les fondements et le développement du modèle suédois de façon à mieux comprendre ce qui fait la spécificité de ce modèle et son succès, mais aussi à éclairer les orientations actuelles au niveau européen autour de la perspective d'investissement social. Dans un premier temps, les fondements du modèle suédois sont retracés, puis sont analysées les évolutions en présentant la logique et les objectifs ayant soustendu les politiques menées afin de montrer les dimensions essentielles de ce modèle et leurs cohérences. Dans un deuxième temps, ce « modèle social productif » est étudié au regard des résultats obtenus en matière économique et sociale. En guise de conclusion, le modèle social productif suédois est comparé à la perspective d'investissement social telle qu'elle s'est développée en Europe (sous l'impulsion notamment de la politique de la Troisième Voie britannique), de façon à souligner les points communs mais aussi les divergences qui marquent ces approches.

\section{Les fondements et caractéristiques principales du modèle suédois}

Si les prémices de l'État-providence suédois remontent au XIXe siècle, $c^{\prime}$ est au début du $X X^{e}$ siècle que se développent les premières assurances sociales et que les éléments fondateurs du modèle se mettent en place. Les sociauxdémocrates, qui arrivent au pouvoir pour la première fois dans les années 1920, s'emparent de la question sociale, celle-ci devenant le pilier central de la politique sociale-démocrate, à tel point que l'histoire de l'État-providence et celle du Parti social-démocrate demeurent indissociables (Korpi, 1983 ; Esping-Andersen, 1992). Quelques personnages ont joué un rôle majeur dans la formation de l'État-providence suédois, déterminant les caractéristiques institutionnelles à partir desquelles il a continué de se développer pendant tout le XXe siècle et qui font, aujourd'hui encore, la spécificité du modèle. Alva et Gunnar Myrdal ainsi que Gustav Möller sont ainsi généralement reconnus comme les architectes principaux de I'État-providence suédois (Rothstein, 1985 et 1998 ; Hirdman, 1989), les premiers ayant pensé la dimension productiviste et $d^{\prime}$ investissement social du modèle ainsi que sa politique familiale très progressive, le second ayant formé le caractère universel du modèle autour de services de qualité sur lesquels reposent tant la légitimité du système (Rothstein, 1998 ; Morel, 2007) que sa capacité à fortement réduire les inégalités (Korpi et Palme, 1998).

\section{Une politique sociale productive et prophylactique}

Dans les années 1930, dans un contexte de crise économique et de crise démographique importante, A. et G. Myrdal, deux éminents sociauxdémocrates (3), développent une nouvelle vision pour la politique sociale, orientée vers I'organisation efficace de la production et de la reproduction, et visant une "transformation radicale » de la société. Leurs idées figurent dans de nombreux rapports et ouvrages, le plus célèbre étant le livre de 1934 intitulé Kris i befolkningsfrågan [Crise dans la question de la population]. Dans ce dernier, ils utilisent les préoccupations démographiques de l'époque pour argumenter en faveur $d^{\prime}$ 'une politique sociale ambitieuse et radicale. Retournant les préoccupations des conservateurs autour de la quantité et de la qualité de la population, ils mettent en avant l'argument selon lequel la baisse de la fécondité est due à des difficultés socio-économiques provoquées par I'industrialisation et l'urbanisation rapide. Les enfants ne sont plus considérés par les familles comme une force de travail supplémentaire pour l'exploitation agricole, mais comme un coût supplémentaire pour les ménages et une charge supplémentaire dans des logements surpeuplés. Pour A. et G. Myrdal, il était donc nécessaire de renforcer l'aide aux familles, notamment par une amélioration des logements et l'instauration d'aides au logement, mais aussi par le biais de politiques soutenant le travail des femmes - le modèle de famille à deux revenus étant censé protéger les familles de la pauvreté - tout en leur permettant de réaliser leur fécondité souhaitée. Mais pour les Myrdal, augmenter la fertilité ne devait pas être un but en soi. Plus important que la «quantité » de la population devait être sa « qualité ». Ici, les Myrdal, adressant les préoccupations eugénistes des conservateurs qui craignaient que les aides aux familles ne profitent d'abord aux enfants des couches populaires de "qualité inférieure », soutiennent que la "qualité des enfants» n'est pas déterminée biologiquement mais qu'elle est liée à des facteurs socio-économiques et à l'éducation (Appelqvist, 2007). Ainsi était-il nécessaire, si on voulait garantir la "qualité » de la population, de mettre en œuvre un vaste ensemble de politiques telles que le développement de services d'accueil pour les jeunes enfants, le développement des services d'éducation et de santé, des politiques de soutien aux familles et à l'emploi des femmes, qui permettraient $d^{\prime}$ investir dans le capital humain de la nation.

(3) G. Myrdal a par ailleurs reçu le prix Nobel d'économie en 1974, et A. Myrdal le prix Nobel de la paix en 1982. 
Si les Myrdal abordaient les préoccupations démographiques de leur époque, dans un contexte de crise économique, leur argument était également fortement lié à une préoccupation concernant la croissance économique et la productivité : sans une population en bonne santé et éduquée qui se reproduit, la productivité de l'économie ne peut être soutenue. Les politiques sociales proposées étaient ainsi présentées non seulement comme un moyen de garantir la sécurité individuelle, la redistribution des richesses et la reproduction de la population, mais aussi, et surtout, comme une façon de promouvoir une organisation plus efficace de la production. G. Myrdal utilise alors le terme de "politique sociale productive» pour désigner cette nouvelle conception du rôle de la politique sociale pour l'économie. Avec ce terme, G. Myrdal souligne que les effets productifs des ressources consacrées à la politique sociale peuvent être comparés aux effets productifs des ressources consacrées aux activités productives. Cette nouvelle conception de l'interaction entre politique sociale productive et croissance économique va rapidement devenir l'un des éléments fondamentaux du modèle suédois, et caractériser la perspective réformiste qui distingue la socialdémocratie du socialisme et du marxisme (Andersson 2006 ; Berman, 2006).

Cette nouvelle conception se veut pragmatique et s'oppose à la politique sociale alors en place qui est décriée comme étant: «[...] superficielle[...] elle soigne les symptômes mais ne traite pas le mal à la racine. On donne l'assistance aux pauvres, des aides au chômage aux chômeurs, on met les malades dans des hôpitaux, les alcooliques dans des maisons pour alcooliques, les fous dans des asiles, etc. mais on fait infiniment peu pour éviter que ces phénomènes se produisent " (Myrdal, 1932:24, traduction de l'auteure). Pour A. et G. Myrdal, il s'agit de donner une nouvelle direction à la politique sociale afin de traiter les causes sous-jacentes des problèmes sociaux. Ainsi, selon eux, «la politique de population [proposée...] se révélera, dans la pratique, être tout simplement une intensification du rôle important de la politique sociale envers la famille et les enfants. Une telle politique sociale est prophylactique et préventive, et non pas simplement symptomatique ou curative. Au début, la politique sociale doit, par nécessité, être restreinte à la phase curative. On doit d'abord s'occuper de ceux qui sont dans le besoin: les pauvres, les malades, les vieux, les alcooliques, les prostituées, les criminels, et tous les autres groupes sociopathologiques. Cela est un devoir social. Mais aujourd'hui la politique sociale suédoise est déjà plutôt bien développée et a traversé sa phase initiale. De fait, la politique curative, bien qu'elle ne soit pas encore arrivée à maturité, est du moins déjà en assez bonne voie.
Il est donc tout à fait logique que nous nous trouvons maintenant à l'aube d'une ère nouvelle dans la politique sociale, où nous nous efforcerons non seulement d'aider ceux dans la détresse et de guérir les maux, mais aussi de les prévenir. Une telle politique préventive doit naturellement être dirigée vers les familles et les enfants. Il s'agit là dans une très large mesure d'un "investissement" dans le capital personnel du pays. Cela peut donc être défendu non seulement sur la base de la charité et de la justice, comme l'ancienne forme de politique sociale, mais aussi sur la base de la gestion du capital humain dans l'intérêt de l'économie nationale » (Myrdal, 1938 a, traduction de I'auteure). Lors d'une conférence donnée en 1938 à I'Economic Society à Copenhague, G. Myrdal souligne, par ailleurs, qu'une telle approche de la politique sociale a un coût que I'on ne doit pas nier, mais que, contrairement au coût des politiques sociales traditionnelles, les politiques envers les familles et les enfants doivent être conçues comme "un investissement : un investissement dans la santé et la capacité de travail de la génération montante, c'est-à-dire de la population future. Cet investissement dans le capital personnel peut, du point de vue des valeurs sociopolitiques pertinentes, être aussi profitable, voire plus profitable, qu'un investissement dans le capital réel "mort". [...] nous devons de fait cesser de considérer les coûts de la politique sociale simplement comme des coûts de consommation improductifs constants. Ces coûts peuvent être des investissements dans ce qui représente au plus haut degré une consommation sociale "productive"» (Myrdal, 1938 b, traduction de l'auteure).

L'accent est ainsi mis sur les familles, et en particulier sur les enfants, ces derniers représentant la main-d'œuvre future sur laquelle reposera la prospérité économique du pays, mais aussi ceux par qui la transformation radicale de la société pourra advenir, I'objectif étant l'abolition des classes sociales, non pas par le renversement de la bourgeoisie par le prolétariat mais par l'amélioration des conditions de la classe ouvrière et l'élimination des barrières sociales. La mise en place de structures d'accueil de qualité pour la socialisation précoce des jeunes enfants est ainsi présentée comme une mesure permettant de garantir l'égalité des chances pour tous les enfants, quel que soit le milieu familial.

Des services universels de qualité pour offrir les mêmes capabilités à tous

Cette idée d'une politique sociale productive, capable de construire une société efficace économiquement tout en rendant possible une transformation sociale radicale, va trouver un écho auprès de Gustav Möller, ministre des Affaires sociales de 1932 à 1951. Ce dernier souhaite mettre en place 
une bureaucratie sociale moderne, basée sur un principe $d^{\prime}$ " universalisme » afin de simplifier la gestion de l'aide sociale et d'ôter les stigmates attachés à celle-ci, mais aussi de façon à faire bénéficier toute la population, riches et pauvres, de mêmes prestations de qualité. De même que les époux Myrdal, G. Möller marque sa préférence pour des prestations offrant les capabilités nécessaires aux individus pour assurer leur autonomie mais aussi pour soutenir leur productivité, son idée étant que seule la croissance économique peut permettre une redistribution des richesses, et non la confiscation des richesses des plus aisés (Tilton, 1990). L'accent est alors mis sur le développement de services publics universels de façon à soutenir les individus tout au long de leur vie. Ce choix de mettre en place des services publics plutôt que de développer des prestations en espèces vise également à pallier les défaillances du marché. En effet, le fait d'offrir des prestations en espèces ne garantit nullement que le marché réponde convenablement à la demande, et ne permet pas de garantir un même accès et une même qualité de services à toute la population.

Le principe avancé par G. Möller sera d'offrir des services identiques pour tous mais de qualité élevée pour que ne se crée pas une demande pour des services alternatifs privés chez les plus riches (Tilton, 1990). En incluant pauvres et riches dans un même système de services publics de qualité, il s'agissait d'éradiquer les inégalités de traitement liées aux inégalités sociales non pas en focalisant I'aide sur les plus démunis, mais en s'assurant le soutien des classes moyennes et aisées en les incluant dans des programmes d'assurance et des services publics universels et de qualité. Cette volonté d'inclure les classes moyennes et aisées dans un système unique deviendra une stratégie politique centrale de la part des sociaux-démocrates à partir des années 1950, avec la mise en place, au sein du système universel d'assurance sociale, d'indemnités proportionnelles au revenu pour éviter que les plus aisés ne mettent en place leurs propres systèmes d'assurance privée (Svensson, 1994).

Ce point est essentiel pour comprendre l'Étatprovidence suédois et sa forte capacité redistributive. En effet, le système repose sur un apparent paradoxe : au lieu de focaliser l'aide sur les plus démunis, l'État-providence suédois redistribue les ressources à toute la population, et proportionnellement plus aux plus aisés qu'aux plus démunis. Pourtant, loin de constituer une dilution des ressources, une telle stratégie permet, au contraire, une plus forte redistribution et, surtout, une plus grande égalisation des conditions et des chances, en tirant vers le haut ceux qui sont le moins bien dotés. Ce «paradoxe de la redistribution », qui a été très bien analysé par Walter Korpi et Joakim
Palme (1998), tient au fait qu'en incluant toute la population dans un même système universel généreux, on augmente le consentement des plus riches à contribuer au système, permettant ainsi d'augmenter la masse des ressources à redistribuer et de garantir la légitimité du système en donnant la possibilité aux plus démunis de bénéficier non pas simplement d'un filet de sécurité au plus bas niveau, mais de prestations de qualité qui satisfont également les classes moyennes et aisées. Pour G. Möller, un tel système universel permet non seulement une plus forte redistribution des ressources, mais offre aussi un meilleur rapport coût-efficacité puisqu'il réduit les coûts élevés liés au contrôle des ressources ainsi que les coûts de gestion associés à des assurances privées multiples.

Si la conception de G. Möller diverge de celle des époux Myrdal concernant la façon d'aider les familles (son approche universaliste - mise en place d'allocations familiales pour tous indépendamment des ressources - étant moins paternaliste que celle des Myrdal qui souhaitaient mettre en place une aide en nature aux familles les plus démunies de façon à contrôler le bon usage de I'aide allouée), G. Möller partage néanmoins avec les Myrdal I'idée que les enfants représentent la richesse la plus précieuse de la société, et qu'ils doivent être protégés et éduqués avec soin (Tilton, 1990:15). Cette idée de l'investissement dans la petite enfance comme un investissement productif va devenir profondément ancrée dans l'Étatprovidence suédois. Ainsi peut-on lire dans un rapport public d'enquête de 1951, concernant le développement des crèches, qu'une des raisons pour laquelle la société doit accroître sa responsabilité dans la prise en charge des jeunes enfants est que "les dépenses qu'engage la société pour les crèches et la garde des enfants représentent un investissement productif, un investissement dans notre patrimoine le plus important et le plus précieux: les enfants " (SOU 1951:15, p. 158, traduction de l'auteure).

À partir des années 1970, lorsque les services d'accueil pour les jeunes enfants commencent véritablement à se développer, I'objectif d'investir dans le capital humain futur de la nation et de donner les mêmes chances à tous les enfants indépendamment de leur milieu familial se double d'un autre objectif : tirer profit du capital humain des mères en leur apportant le soutien nécessaire à leur investissement productif sur le marché du travail (Morel, 2007 et 2008). Dans un contexte de pénurie de main-d'œuvre, il s'agit à la fois d'inciter les femmes à s'investir et à se maintenir sur le marché du travail, d'autant plus qu'elles représentent une main-d'œuvre mieux formée que la maind'œuvre immigrée en raison d'une forte augmentation du niveau d'études chez les femmes dans la 
période d'après-guerre. Mais il s'agit aussi, selon le rapport pour l'égalité rédigé en 1969 par Alva Myrdal pour le parti social-démocrate et la Confédération des syndicats LO (Landsorganisationen), de promouvoir un modèle familial à deux revenus, considéré comme le meilleur moyen d'aller vers une individualisation totale des droits sociaux et comme un rempart efficace contre la pauvreté des familles, notamment celles avec enfants. Dans ce rapport est affirmé le fait que le revenu de son propre travail, ainsi qu'un système de protection sociale moderne et individualisé, sont les deux pierres angulaires sur lesquelles doit reposer la sécurité de l'individu, et non pas le lien conjugal ou familial (Myrdal, 1971). Il s'agit, enfin, d'augmenter et de maximiser les taux d'emploi de l'ensemble de la population afin d'accroître l'assiette fiscale et pouvoir ainsi extraire les ressources nécessaires au maintien d'un État-providence fort.

\section{Une politique d'emploi active}

La stratégie d'investissement social trouvera également son expression dans le modèle économique mis en place dans les années 1950, le fameux modèle "Rehn-Meidner », du nom de ses deux concepteurs Gösta Rehn et Rudolf Meidner, tous deux économistes à la Confédération des syndicats LO. C'est à la fin des années 1940 que G. Rehn et R. Meidner commencent à réfléchir aux problèmes $d^{\prime}$ inflation que risque $d^{\prime}$ engendrer la politique économique keynésienne alors en vigueur. Selon eux, la politique keynésienne est certes utile en période de récession, mais la période d'après-guerre est caractérisée, en Suède, par une forte croissance (jusqu'à $7 \%$ par an) et un très faible chômage (entre 1 et $2 \%$ ) (Benner et Bundgaard Vad, 2000). Les syndicats sont alors en position de force pour demander des hausses de salaire, risquant ainsi d'engendrer une forte inflation, ce qui, à terme, ferait augmenter le chômage. Une politique monétaire restrictive n'est pas non plus souhaitable si I'on veut maintenir le plein emploi. G. Rehn et R. Meidner souhaitent ainsi remplacer la politique keynésienne de «stop and go » (I'alternance de politiques monétaires tour à tour expansionnistes ou restrictives) par une politique permettant de garder l'inflation sous contrôle de façon permanente (Erixon, 2000). Mais leur objectif n'est pas seulement de maintenir à la fois le plein emploi et la stabilité des prix, ils souhaitent, en outre, combiner croissance et équité. Pour Lennart Erixon, c'est notamment en cela que le modèle "Rehn-Meidner » est véritablement unique, les modèles macro-économiques modernes ne s'attachant au mieux qu'à deux objectifs à la fois, généralement la stabilité des prix et le plein emploi (Erixon, 2000). La solution proposée par G. Rehn et R. Meidner pour résoudre cette quadrature du cercle comporte ainsi plusieurs volets.
Il s'agit, d'une part, d'accroître la productivité en forçant les entreprises les moins productives à disparaître ou à se rationaliser. C'est par le biais $d^{\prime}$ une politique salariale solidaire que les entreprises les moins productives seraient conduites à disparaître. En effet, l'idée de G. Rehn et R. Meidner était d'amener les syndicats à négocier, pour chaque branche, un même salaire pour un même travail, tirant ainsi les salaires vers le haut, mais en obtenant néanmoins de la part des syndicats que cette hausse se fasse de façon solidaire afin d'obtenir une certaine compression des salaires entre les différentes branches. L'objectif était $\mathrm{d}^{\prime}$ obtenir une certaine modération salariale dans les secteurs les plus productifs pour éviter les tendances inflationnistes. Cette hausse des salaires ferait en sorte que les entreprises les moins productives seraient contraintes de fermer. Dans un même temps, comme la hausse des salaires serait modérée, ces salaires solidaires resteraient en deçà de ce que les entreprises les plus productives seraient à même de payer, leur permettant ainsi de dégager plus de profits, qu'elles pourraient ainsi réinvestir. Cela encouragerait également la création de nouvelles entreprises dans des secteurs plus dynamiques.

Puisqu'il s'agissait néanmoins de maintenir le plein emploi, il fallait, d'autre part, développer une politique d'emploi active permettant de reformer et de réorienter la main-d'œuvre ainsi dégagée par la fermeture des entreprises les moins productives vers les entreprises les plus productives et vers de nouveaux secteurs pour lesquels il manquait de la main-d'œuvre. Toutefois, de façon à ce que les travailleurs ne portent pas seuls le coût des restructurations économiques, cette politique active d'emploi comprenait, outre l'offre de reformation, une aide à la délocalisation et une indemnité chômage généreuse pour assurer la transition. En accélérant les restructurations et la croissance, ce mécanisme a contribué à l'augmentation rapide du niveau de vie dans les décennies d'après-guerre, permettant également une augmentation de l'imposition afin de financer l'expansion de services sociaux universels et de qualité, bénéficiant à toute la population.

Ainsi, si l'accent est mis sur la mise en œuvre de politiques visant à développer le capital humain, la préservation de ce dernier, par le biais de politiques actives d'emploi, est considérée comme tout aussi importante. De plus, il ne s'agit pas simplement $d^{\prime}$ " activer » la main-d'œuvre par le biais de mesures coercitives, mais aussi de protéger le capital humain par le biais d'indemnisations généreuses en cas de chômage ou de maladie. Le fait de garantir la sécurité du revenu en période de chômage était perçu comme un élément essentiel pour aider les travailleurs à surmonter la peur du changement et des restructurations économiques 
nécessaires. Comme l'a souligné G. Esping-Andersen, par le biais de cette politique sociale productive, la social-démocratie suédoise est parvenue à réconcilier les deux objectifs d'égalité et d'efficacité qui avaient jusque-là été compris comme des objectifs contradictoires: "l'égalité n'a pas été promulguée comme étant simplement compatible avec l'efficacité. Elle est devenue, au contraire, une condition préalable à son optimisation : un pouvoir d'achat plus équitablement distribué est une condition sine qua non pour la performance macro-économique; la politique familiale est un investissement dans le capital humain futur; l'égalisation des ressources, comme la santé ou l'éducation, est le fondement d'une productivité du travail optimale; la politique salariale solidaire et les politiques actives d'emploi stimulent la modernisation industrielle ; la sécurité du revenu permet de surmonter la résistance naturelle des travailleurs à la rationalisation économique ; et la politique sociale préventive diminue la perte de capital humain et les coûts économiques »(EspingAndersen, 1992:38).

Si le modèle "Rehn-Meidner » est progressivement abandonné vers la fin des années 1970, un de ses éléments centraux va néanmoins perdurer dans les années 1990 et 2000 : I'accent mis sur les mesures favorisant la mobilité professionnelle par le biais de la formation. Notamment, en période de crise économique sévère dans les années 1990, la Suède mettra en place un programme ambitieux de formation tout au long de la vie et renforcera son engagement dans l'investissement dans le capital humain dès le plus jeune âge.

\section{Une stratégie d'investissement dans le capital humain}

Le début des années 1990, qui est marqué par une crise économique aiguë en Suède, amène le gouvernement de droite (1991-1994), puis socialdémocrate, à prendre des mesures d'assainissement budgétaire drastiques. Le gouvernement socialdémocrate (de retour au pouvoir en 1994) va néanmoins poursuivre une stratégie explicite d'investissement dans le capital humain de façon à relancer la croissance. Ainsi, pendant la seconde moitié des années 1990, le gouvernement introduit une série de réformes qui réduisent la générosité des prestations sociales : baisse de $10 \%$ du taux de remplacement pour l'assurance maladie, pour l'assurance chômage, et pour le congé parental ; diminution du montant des allocations familiales ; réforme des retraites, etc. Mais ces coupes vont épargner en partie les services sociaux, en particulier les services de santé et les services d'accueil à la petite enfance. En effet, lors de la campagne électorale de 1994, les sociaux-démocrates argumentent qu'il sera toujours possible de réaugmenter les prestations en espèces si la conjoncture économique s'améliore, alors que des services de santé, d'accueil des jeunes enfants ou d'éducation de mauvaise qualité risqueraient de porter préjudice aux enfants de manière irréversible (SAP, 1994 ; Palme et Wennemo, 1998) et, par là même, d'affecter la croissance économique future du pays.

Ce souci d'une main-d'œuvre de qualité pour le futur se renforce davantage encore dans un contexte de compétition mondiale dans laquelle la Suède, comme les autres pays nordiques, cherche à développer un avantage compétitif en promouvant une économie de la connaissance dans laquelle le capital humain, constitué de capacités cognitives et non cognitives, devient une valeur très importante. Ainsi, lors de sa déclaration gouvernementale au Parlement en 1996, le Premier ministre Göran Persson déclare que : "I'apprentissage tout au long de la vie doit être une pierre angulaire de la politique gouvernementale de lutte contre le chômage. La Suède doit être en mesure de rivaliser par le biais de compétences élevées, et les conditions préalables à cela sont à fournir par le biais d'une qualité élevée dans toutes les formes d'éducation, de l'accueil préscolaire à l'enseignement supérieur. L'accueil préscolaire doit contribuer à l'amélioration des premières années importantes de la scolarité obligatoire» (Göran Persson, cité dans Korpi, 2007:65, traduction de I'auteure). Un vaste programme de "développement de la connaissance » (Kunskapslyft) est mis en œuvre à partir de 1996 pour une période de cinq ans, visant à relever le niveau d'éducation de toute la population, en offrant la possibilité aux moins qualifiés de compléter un diplôme du secondaire et, aux chômeurs, de bénéficier de nouvelles formations tout en recevant des prestations. Des aides financières et des aides pédagogiques spécifiques ont également été mises en place pour aider les personnes avec des difficultés particulières. L'objectif poursuivi était une augmentation globale du niveau de formation afin de donner à l'ensemble de la population les capacités nécessaires pour pouvoir participer à l'éducation tout au long de la vie, le but étant de promouvoir la croissance par une augmentation de la productivité et des salaires, et de transformer la Suède en une économie de la connaissance (SOU 1996:27).

Cet investissement dans l'éducation et la formation se retrouve à tous les niveaux. La Suède se démarque particulièrement par ses taux de préscolarisation élevés, en particulier pour les enfants âgés de moins de 3 ans, avec $76 \%$ des enfants de 1 an à 3 ans (4)

(4) Jusqu'à l'âge de un an, les enfants sont gardés par leurs parents par le biais d'un congé parental bien rémunéré. 
fréquentant un mode de garde formel, ainsi que $95 \%$ des enfants de 4 ans et 5 ans en 2011 (5), soit bien au-delà des objectifs fixés au niveau européen. Il s'agit, en outre, de services d'accueil de qualité, avec un personnel qualifié et un taux $d^{\prime}$ encadrement $d^{\prime}$ un adulte pour six enfants. Cet investissement important dans l'éducation des jeunes enfants se poursuit également tout au long de la vie. Ainsi la Suède consacrait-elle $7,3 \%$ de son produit intérieur brut à l'éducation (du préscolaire au tertiaire) en 2009, contre une moyenne, pour les pays de l'OCDE, de 5,8\% $(5,9 \%$ pour la France). Surtout, la Suède se classe en première position en ce qui concerne la formation continue, et ce quel que soit le niveau de qualification initial des individus : $72 \%$ des 25-34 ans et $60 \%$ des 55-64 ans avaient participé à une formation en 2008, contre une moyenne, pour les pays de I'OCDE, de $37 \%$ et $23 \%$ respectivement (OCDE, 2012).

En ce qui concerne le niveau de qualification de la population, $87 \%$ des personnes âgées de 24 ans à 65 ans ont au moins atteint l'enseignement secondaire supérieur, contre $74 \%$ dans les pays de I'OCDE. Cet écart est particulièrement marqué au sein de la cohorte la plus âgée. Alors que $91 \%$ des 24-35 ans ont au moins atteint I'enseignement secondaire supérieur (la moyenne de I'OCDE est de $82 \%$ ), $77 \%$ des 55-64 ans en Suède ont atteint ce niveau d'enseignement, notamment grâce à l'éducation pour les adultes, ce qui représente quinze points de plus que la moyenne de I'OCDE (OCDE, 2012). La Suède a, par ailleurs, déjà atteint l'objectif, fixé par l'Union européenne dans sa stratégie Europe 2020, de faire en sorte qu'au moins $40 \%$ des 30-34 ans aient une formation de niveau tertiaire : près de $42 \%$ des 25-34 ans sont titulaires d'un diplôme du tertiaire (contre une moyenne de $38 \%$ pour I'OCDE). Parmi l'ensemble de la population âgée de 25 ans à 64 ans, $34 \%$ ont atteint ce niveau d'enseignement, soit trois points de plus que la moyenne de l'OCDE (OCDE, 2012). Enfin, avec un taux de $10 \%$ (contre $16 \%$ pour l'OCDE, $17 \%$ pour la France), la Suède présente un des plus faible pourcentage de jeunes de 15 ans à 29 ans se trouvant ni en emploi, ni en éducation ou formation (les NEET) (OCDE, 2012).
Pour terminer, il convient également de noter le poids relativement plus faible du milieu familial sur le niveau d'étude des enfants, la Suède faisant partie des pays de l'OCDE dans lesquels le taux de reproduction des inégalités scolaires est le moins marqué. De même, les différences de performance, mesurées par les tests PISA (6), entre les enfants fréquentant des écoles dans des quartiers plus défavorisés et ceux scolarisés dans des quartiers plus favorisés sont-elles sensiblement plus faibles que pour la moyenne des pays de l'OCDE (OCDE, 2012).

\section{Quelles conséquences des politiques menées?}

Dans la mesure où les politiques mises en œuvre visent à permettre de concilier équité sociale et efficacité économique, il convient à présent d'évaluer le succès du modèle dans la réalisation de ce double objectif. Tout d'abord, on peut observer que les pays nordiques font partie des pays dans lesquels les inégalités de revenu sont les plus faibles (un coefficient de Gini (7) autour de 2,4 contre une moyenne européenne de 3,1 en 2011 (données Eurostat). Il s'agit, en outre, de pays dans lesquels la mobilité sociale est relativement forte, ces pays présentant les plus faibles taux de reproduction des inégalités d'éducation et de revenus (Esping-Andersen, 2009 ; Corak, 2012 ; Kvist et al., 2012), même si les inégalités se sont accrues au cours des deux dernières décennies (mais partant d'un niveau très bas, celles-ci restent encore faibles d'un point de vue comparatif). Dans un même temps, les pays nordiques sont classés parmi les pays les plus compétitifs selon le rapport sur la compétitivité mondiale (Global Competitiveness Report, GCR), rédigé annuellement en marge du Forum économique mondial de Davos, la Suède présentant un indice mondial de la compétitivité (Global Competitiveness Index, GCI) qui la place parmi les cinq pays les plus compétitifs au monde depuis de nombreuses années (8). Les pays nordiques présentent également des taux d'emploi élevés, le taux d'emploi global en Suède se situant à $81,5 \%$, ce qui représente le taux d'emploi le plus élevé de l'OCDE derrière I'Islande. De plus, ces taux d'emploi élevés se retrouvent

\footnotetext{
(5) Données de l'Agence nationale pour l'éducation (Skolverket) : http://www.skolverket.se/statistik-och-analys.

(6) PISA est une enquête menée tous les trois ans auprès de jeunes de 15 ans dans les 34 pays membres de l'OCDE. Elle évalue l'acquisition de savoirs et savoir-faire au terme de la scolarité obligatoire. Les tests portent sur la lecture, la culture mathématique et la culture scientifique.

(7) Le coefficient de Gini est une mesure du degré d'inégalité de la distribution des revenus dans une société donnée. Il est compris entre 0 (égalité parfaite) et 10 (inégalité absolue).

(8) L'indice mondial de la compétitivité est un indice composite résultant d'une moyenne pondérée de cent dix variables très diverses (indicateurs macroéconomiques, infrastructures, qualité du système éducatif, situation sociale, intensité de la recherche et du développement, etc.). Cette accumulation de données doit permettre de prendre en compte tous les " facteurs permettant aux économies nationales d'atteindre une croissance économique soutenue et une prospérité à long terme ». Les rapports peuvent être consultés sur le site du World Economic Forum : http://www.weforum.org/issues/globalcompetitiveness
} 
à tous les niveaux de qualification, y compris pour les moins qualifiés (en dessous du niveau secondaire) qui ont un taux d'emploi autour de $67 \%$, contre $54 \%$ en moyenne pour les pays d'Europe continentale (OCDE, 2012). II semblerait que ce résultat soit lié au niveau moyen de compétences cognitives que possèdent les personnes peu qualifiées. De fait, s'il existe partout une pénalité en termes d'emploi pour les moins qualifiés, cette pénalité est plus forte dans les pays où les personnes peu qualifiées ont les plus bas niveaux de compétences cognitives (États-Unis, Europe continentale et Europe du Sud). La pénalité est plus faible dans les pays tels que les pays nordiques, où les capacités cognitives des moins qualifiés sont plus élevées (Abrassart, 2012). Le fait que les capacités cognitives des moins qualifiés soient plus élevées dans les pays nordiques semble à son tour être lié à quatre éléments clés : des inégalités sociales et économiques plus faibles; l'effet positif exercé par la préscolarisation précoce des jeunes enfants dans des structures de qualité sur la réussite scolaire à long terme; un système éducatif bien plus fortement égalitaire que dans les autres pays ; et un investissement important dans l'éducation pour les adultes et la formation continue (EspingAndersen, 2009 ; Nelson et Stephens, 2012 ; Lundvall et Lorenz, 2012).

Les analyses de Moira Nelson et John D. Stephens montrent, par ailleurs, qu'il y a un impact significatif des politiques menées d'investissement social dans le capital humain tant sur la quantité d'emplois créés que sur la qualité des emplois, avec notamment un effet particulièrement concentré dans la partie inférieure de la distribution des compétences (Nelson et Stephens, 2012). Comme le soulignent également Jon Erik Dølvik, Jørgen Goul Andersen et Juhhana Vartiainen (2012), les politiques menées depuis les années 1990 ont stimulé un transfert vers de l'emploi davantage qualifié avec une plus forte valeur ajoutée, ce qui se traduit aujourd'hui par une part de I'emploi peu rémunéré comparativement plus faible que dans les autres pays. Ces politiques ont également permis à ces pays de résister relativement mieux à la crise. Les analyses de Bengt-Åke Lundvall et Edward Lorenz montrent l'impact positif des politiques menées ainsi que l'importance d'un système éducatif égalitaire et d'inégalités sociales et économiques plus faibles sur la forte capacité d'innovation des pays nordiques (Lundvall et Lorenz, 2012). Ainsi, les pays nordiques semblent démontrer que, non seulement il n'y a pas de compromis nécessaire entre équité sociale et efficacité économique mais aussi que l'équité est même une précondition pour cette dernière. Il convient également de noter les taux d'emploi des femmes très élevés (autours de $70 \%$ ) dans ces pays, qui ne les empêchent pas de maintenir une fécondité supérieure à la moyenne européenne avec un indice de fécondité autour de 1,9 (données Eurostat 2011). Là encore, ces bons résultats semblent tenir aux politiques sociales menées pour promouvoir la conciliation vie familiale-vie professionnelle (congé parental bien rémunéré, garantie d'une place en structure d'accueil préscolaire de bonne qualité et à un coût très faible) mais également aux efforts qui ont été menés pour atteindre une meilleure égalité entre les sexes (Esping-Andersen, 2009).

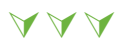

\section{Deux conceptions de l'investissement social en Europe}

La stratégie d'investissement social est devenue un concept à la mode au niveau européen depuis la fin des années 1990, cette notion $d^{\prime}$ « investissement social » ayant notamment soutenu l'agenda de Lisbonne que I'Union européenne a adopté en 2000 dans le but de faire de l'Europe "l'économie de la connaissance la plus compétitive et la plus dynamique du monde, capable d'une croissance économique durable accompagnée d'une amélioration quantitative et qualitative de l'emploi et d'une plus grande cohésion sociale »(Conseil européen, 2000). Si cette perspective semble s'inspirer des idées concernant le potentiel productif de la politique sociale telles qu'elles ont été développées en Suède dès les années 1930, comme d'autres analyses l'ont noté (Morel et al., 2012 b) la stratégie européenne est également, voire essentiellement, très fortement associée à la politique de la Troisième Voie du parti travailliste britannique sous Tony Blair et Gordon Brown, et inspirée par les travaux d'Anthony Giddens.

De fait, afin d'éclairer les orientations européennes actuelles, il paraît utile de souligner la spécificité de l'approche sociale-démocrate telle qu'elle a été mise en œuvre en Suède, par rapport à l'approche que l'on retrouve en Europe, plus inspirée par la Troisième Voie britannique. Un premier point de divergence important qui mérite d'être souligné est que, là où la stratégie d'investissement social de la Troisième Voie met l'accent sur les obligations liées à la citoyenneté sociale et sur la responsabilité individuelle, la stratégie suédoise insiste, au contraire, sur les effets productifs des droits sociaux de la citoyenneté sociale. De même, alors que, dans la version de la Troisième Voie, il s'agit de réduire les sécurités pour promouvoir le changement, la stratégie suédoise met, à l'inverse, I'accent sur la protection, l'idée étant qu'on ne peut promouvoir le changement sans, en même temps, assurer la sécurité des individus, l'insécurité étant perçue comme une source $d$ 'inefficacité et comme une entrave à la croissance. Pour 
l'ex-Premier ministre suédois G. Persson, la sécurité crée des individus courageux qui " osent dépenser, être créatifs, critiquer et avoir des idées sur leur lieu de travail, étudier et élever une famille » (cité dans Andersson, 2007:304), la sécurité individuelle est ainsi une condition essentielle pour assurer la croissance, la productivité et la compétitivité. La notion $d^{\prime}$ « égalité » est également perçue très différemment. Alors que l'égalité est considérée comme un élément central pour la poursuite de l'efficacité économique et que la réduction des inégalités est un objectif explicite de la politique sociale productive sociale-démocrate, A. Giddens estime que la quête de l'égalité est l'erreur historique de la "vieille gauche » (Morel et al., 2012 b).

Enfin, le rôle assigné à la politique sociale diffère. Conçu pour agir comme un «tremplin » pour le changement dans I'approche de la Troisième Voie (le « ressort» provenant tant de l'investissement dans le capital humain que dans les fortes incitations (négatives) en faveur de l'activation), dans la variante sociale-démocrate, la politique sociale est conçue pour apporter aux individus à la fois les capabilités et les incitations nécessaires, mais aussi la sécurité nécessaire pour accompagner l'évolution des besoins de l'économie. C'est donc sur trois piliers que repose le modèle social suédois : la promotion par l'investissement dans le capital humain, I'activation, et la protection tout au long de la vie. Ainsi, dans la version socialedémocrate, une stratégie d'investissement social qui vise à réconcilier efficacité et équité ne peut se réduire à une simple réorientation des dépenses sociales "passives» vers des dépenses sociales " actives ».

Pour conclure, on peut dire que la politique sociale suédoise est effectivement "démarchandisante " en ce qu'elle assure la sécurité des individus indépendamment de leurs niveaux de ressources (et de leur milieu familial), et elle les protège contre les effets les plus négatifs du marché. Mais il s'agit plus encore d'une politique productive visant à accompagner les besoins du capitalisme, tout en conciliant efficacité économique et équité sociale, l'équité sociale apparaissant comme une condition préalable à l'efficacité économique. 


\section{Références $\mid$ bibliographiques}

- Abrassart A., 2012, Cognitive skills matter: The employment disadvantage of low-educated workers in comparative perspective, European sociological review, first published online March 26, 2012.

- Andersson J., 2007, Solidarity or competition? Creating the European knowledge society, in European Solidarities. Tensions and contentions of a concept [L. Magnusson et B. Stråth (eds)], Bruxelles, PIE Peter Lang:293-309.

- Andersson J., 2006, Between growth and security. Swedish social democracy from a strong society to a third way, Manchester, Manchester University Press.

- Appelqvist Ö., 2007, L'argument démographique dans la genèse de l'État-providence suédois, Vingtième Siècle. Revue d'histoire, vol. 95:15-28.

- Benner M. et Bundgaard Vad T., 2000, Sweden and Denmark: Defending the welfare state, in Welfare and work in the open economy. Volume 2: Diverse responses to common challenges [F. Scharpf et V. Schmidt (eds.)], Oxford, Oxford University Press:399-466.

- Berggren H. et Trägårdh L., 2006, Är svensken människa?: gemenskap och oberoende $i$ det moderna Sverige, Stockholm, Norstedts.

- Berman S., 2006, The primacy of politics. Social democracy and the making of Europe's twentieth century, Cambridge University Press.

- Conseil européen, 2000, "Conclusions de la présidence », Conseil européen de Lisbonne 23 et 24 mars 2000.

- Corak M., 2012, Inequality from generation to generation: The United States in comparison, in The economics of inequality, poverty, and discrimination in the 21st Century [R. Rycroft (ed.)], Santa Barbara, California, ABC-CLIO.

- Dølvik J. E., Goul Andersen J. et Vartiainen J., 2012, «Nordic models in turbulent times developments in social and labour market governance since the crisis in the 1990s», paper presented at the International Sociological Association RC19 annual conference, 23-24 August 2012, Oslo.

- Erixon L., 2000, A Swedish economic policy - The theory, application and validity of the Rehn-Meidner model, Research Papers in Economics, 2000:13.

- Esping-Andersen G., 2009, The incomplete revolution. Adapting welfare states to women's new roles, Oxford, Polity Press.

- Esping-Andersen G., 1992, The making of a social democratic welfare state, in Creating social democracy. A century of the social democratic labor party in Sweden [Misgeld M. et Åmark K. (eds)], Pennsylvania, The Pennsylvania State University Press:35-66.

- Esping-Andersen G., 1990, The three worlds of welfare capitalism, Cambridge, Polity Press.

- Esping-Andersen G., 1985, Politics against markets: the social-democratic road to power, Princeton, Princeton University Press.

- Gautié J., 2003, «Quelle troisième voie ? Repenser I'articulation entre marché du travail et protection sociale », Document de travail, Centre d'études de l'emploi, n 30.

- Hirdman Y., 1989, Att lägga livet till rätta, Stockholm, Carlsson Bokförlag.

- Korpi B. M., 2007, Förskolan i politiken - om intentioner och beslut bakom den svenska förskolans framväxt, Stockholm, Utbildningsdepartementet.

- Korpi W., 1983, The democratic class struggle, Boston, Routledge and Kegan \& Paul.

- Korpi W. et Palme J., 1998, The paradox of redistribution and strategies of equality: welfare state institutions, inequality, and poverty in the Western countries, American sociological review, vol. 63:661-687. 
- Kvist J., Fritzel J., Hvinden B. et Kangas O., (eds.), 2012, Changing social equality: The nordic welfare model in the 21st century, Bristol, The Policy Press.

- Lundvall B-Å. et Lorenz E., 2012, Social investment in the globalising learning economy. A European perspective, in Towards a social investment welfare state? Ideas, policies and challenges [Morel N., Palier B. et Palme J. (eds.), Bristol, Policy Press:235-257.

- Mahon R., 2013, Social investment according to the OECD/DELSA: A discourse in the making, Global Policy, article first published online: 17 janvier 2013, doi: 10.1111/j.1758-5899.2012.00182.x

- Morel N., 2008, Les services d'accueil préscolaire en Suède : entre dispositif de garde d'enfants et dispositif d'éducation, Nordiques, $\mathrm{n}^{\circ}$ 16:27-46.

- Morel N., 2007, «L'État face au social : la (re)définition des frontières de l'État-providence en Suède. Une analyse des politiques de prise en charge des personnes âgées dépendantes et des jeunes enfants de 1930 à 2005 », thèse de sociologie sous la direction de F. Piotet, université Paris I.

- Morel N., Palier B. et Palme J. (eds.), 2012 a, Towards a social investment welfare state? Ideas, policies and challenges, Bristol, Policy Press.

- Morel N., Palier B. et Palme J., 2012 b Beyond the welfare state as we knew it?, in Towards a social investment welfare state? Ideas, policies and challenges [Morel N., Palier B. et Palme J. (eds.)], Bristol, Policy Press:1-30.

- Myrdal A., 1971, Towards equality - the Alva Myrdal report to the Swedish social democratic party, Stockholm, Prisma.

- Myrdal G., 1938 a, Population problems and policies, Annals of the American Academy of Political and Social Science, $\mathrm{n}^{\circ}$ 197:200-215.

- Myrdal G., 1938 b, Conférence prononcée à l’Economic Society à Copenhague le 25 janvier 1938. Extraits publiés dans Ö. Appelqvist et S. Andersson (1998), Gunnar Myrdal. Vägvisare, Stockholm, Norstedts.

- Myrdal G., 1932, Socialpolitikens dilemma, Spektrum 2, nº 4:24-28.

- Myrdal A. et Myrdal G., 1934, Kris i befolkningsfrågan, Stockholm, Albert Bonniers Förlag.

- Nelson M. et Stephens J. D., 2012, Do social investment policies produce more and better jobs?, in Towards a social investment welfare state? Ideas, policies and challenges [Morel N., Palier B. et Palme J. (eds.), Bristol, Policy Press:205-234.

- Organisation de coopération et de développement économiques (OCDE), 2012, Education at a Glance 2012, OECD Indicators, OECD Publishing.

- Palme J. et Wennemo I., 1998, Swedish social security in the 1990s: reform and retrenchment, Välfärdsprojektets skriftserie, Stockholm, The Printing Works of the Cabinet Office and Ministries.

- Rothstein B., 1985, Managing the welfare state: lessons from Gustav Möller, Scandinavian Political Studies, vol. 8, n 3:151-170.

- Rothstein B., 1998, Just institutions matter, Cambridge, Cambridge University Press.

- Sveriges socialdemokratiska arbetareparti (SAP), 1994, "Valmanifest antaget av den socialdemokratiska partistyrelsen », 1994-08-19, Stockholm.

- SOU 1951:15, Daghem och förskolor, Stockholm, Socialdepartementet.

- SOU 1996:27, En strategi för kunskapslyft och livslångt lärande, Stockholm, Utbildnings departementet.

- Svensson T., 1994, Socialdemokratins dominans. En studie av den svenska socialdemokratins partistrategi, Uppsala, Acta Universitatis Upsaliensis.

- Tilton T., 1990, The political theory of Swedish social democracy: Through the welfare state to socialism, Oxford, Clarendon Press. 
